\title{
Epigenetic silencing of microRNA-199a-5p promotes the proliferation of non-small cell lung cancer cells by increasing AKAP1 expression
}

\author{
NENGLI YANG ${ }^{1}$, YAFENG LIANG ${ }^{2}$, TIANQI ZHU ${ }^{1}$, YANXIAO LONG ${ }^{1}$, \\ ZHE CHEN $^{1}$, XUEZHENG ZHANG ${ }^{1}$ and LIUMING JIANG ${ }^{1}$ \\ ${ }^{1}$ Department of Anesthesiology, The First Affiliated Hospital of Wenzhou Medical University; \\ ${ }^{2}$ Department of Pediatric Intensive Care Unit, The Second Affiliated Hospital and Yuying Children's Hospital, \\ Wenzhou Medical University, Wenzhou, Zhejiang 325000, P.R. China
}

Received July 29, 2020; Accepted February 25, 2021

DOI: $10.3892 / \mathrm{ol} .2021 .12695$

\begin{abstract}
MicroRNA (miR)-199a-5p expression is downregulated in a variety of malignancies, including non-small cell lung cancer (NSCLC), and its low expression is associated with a poor prognosis. However, to the best of our knowledge, the mechanism underlying miR-199a-5p downregulation in NSCLC and its target effectors remain to be elucidated. The present study revealed the downregulation of miR-199a-5p expression in NSCLC tissues and cell lines compared with in para-carcinoma tissues and a lung epithelial cell line. Further experiments indicated that the methylation levels of the miR-199a promoter were markedly higher in NSCLC tissues compared with in para-carcinoma tissues. The DNA methyltransferase inhibitor 5-Aza-2'-deoxycytidine markedly increased the expression levels of miR-199a-5p in NSCLC cells. Furthermore, it was identified that miR-199a-5p mimics transfection decreased the expression levels of A-kinase anchoring protein 1 (AKAP1) at both the mRNA and protein levels by targeting the $3^{\prime}$ untranslated region of AKAP1 mRNA. The in vitro experiments demonstrated that miR-199a-5p overexpression inhibited the proliferation and tumorigenicity of NSCLC cells, whereas overexpression of AKAP1 partially recovered the malignant phenotypes, suggesting that AKAP1 may be a downstream effector targeted by miR-199a-5p. Collectively, the present findings indicated that miR-199a-5p may be a novel regulator of AKAP1, and that miR-199a-5p may be a potential tumor suppressor in NSCLC.
\end{abstract}

Correspondence to: Dr Liuming Jiang, Department of Anesthesiology, The First Affiliated Hospital of Wenzhou Medical University, 2 Fuxue Street, Wenzhou, Zhejiang 325000, P.R. China E-mail:a9713030b@163.com

Key words: non-small cell lung cancer, microRNA-199a-5p, hypermethylation, A-kinase anchoring protein 1, proliferation

\section{Introduction}

Lung cancer is the leading cause of cancer-associated mortality worldwide (1). In most countries, for lung cancer patients diagnosed during 2005-2009, the 5-year survival rate ranges between 10 and $20 \%$ (1), which highlights the inadequacy of the current treatment strategies for lung cancer (1). Lung cancer is classified into two main types, small cell lung cancer and non-small cell lung cancer (NSCLC), and NSCLC accounts for $\sim 90 \%$ of all cases (2). In the majority of patients with NSCLC, local or advanced metastasis occurs after diagnosis (3). Therefore, the mechanisms underlying NSCLC development have become a focus of research in recent years.

MicroRNA (miRNA/miR)-199a-5p expression is generally downregulated in tumors and can inhibit the progression of various tumor types, including prostate cancer, breast cancer, hepatocellular carcinoma, ovarian cancer, colorectal cancer and lung cancer, by downregulating different downstream genes (4-9). For example, Hu et al (10) reported that miR-199a-5p could inhibit the migration and invasion of colorectal cancer by targeting discoidin domain receptor-1. In liver cancer, miR-199a-5p inhibits glucose consumption and lactic acid generation by targeting hexokinase 2, thus attenuating the proliferation and tumorigenesis of cancer cells (11). In addition, $\mathrm{Li}$ et al (12) revealed that miR-199a-5p-mediated silencing of ETS proto-oncogene 1, transcription factor represses the invasion of breast cancer cells by decreasing the levels of integrin 1 (12). Additionally, miR-199a-5p has been reported to be a tumor suppressor in NSCLC $(9,13)$. However, the mechanisms of the downregulation of miR-199a-5p expression and the downstream effectors targeted by miR-199a-5p in NSCLC are not fully understood.

A-kinase anchoring protein 1 (AKAP1) is a scaffold protein, which recruits protein kinase A, other signaling proteins and RNA to the outer mitochondrial membrane (14). In hepatocellular carcinoma, high expression levels of AKAP1 are associated with a poor prognosis, suggesting that AKAP1 may serve as a biomarker (15). Another previous study reported that knockdown of AKAP1 sensitizes gastric cancer cells to cisplatin treatment (16). Furthermore, AKAP1 is expressed at 
high levels in lung cancer cells compared with non-tumoral cells, and is associated with the decreased survival rate of patients with lung cancer (17). However, to the best of our knowledge, the mechanisms underlying the aberrant expression of AKAP1 in NSCLC and its cellular function remain unknown.

The present study demonstrated that the hypermethylation of the promoter of miR-199a led to downregulation of miR-199a-5p in NSCLC cells. Exogenous miR-199a-5p suppressed the proliferation and tumorigenicity of NSCLC cells by decreasing AKAP1 expression, which was achieved via direct targeting of the 3' untranslated region (UTR) of AKAP1 mRNA. These findings indicated a novel regulatory mechanism of AKAP1, as well as its role in NSCLC proliferation.

\section{Materials and methods}

Collection of NSCLC tissues. All patients with NSCLC $(n=41$; median age, 61 years; age range, 35-84 years; 14 female and 27 male patients) were diagnosed according to the International Association for Lung Cancer Research 2015 version guidelines (18). NSCLC and paired para-carcinoma tissues $(2 \mathrm{~cm}$ away from the tumor edge) were collected between January 2019 and June 2020 at The First Affiliated Hospital of Wenzhou Medical University (Wenzhou, China). Patients with NSCLC were included that had been diagnosed on the basis of pathological examinations. Histological classification was performed according to WHO Classification of Tumors of the Lung (2015) (19) and tumor staging was performed according to the staging criteria of International Union Against Cancer (UICC, 2017) (20). Patients with NSCLC without radiotherapy or chemotherapy prior to the surgical lung resection were included. Pregnant patients, patients with mental or cognitive impairment and patients with another lung disease were excluded from the present study. Written informed consent was obtained from all patients, and the present study was approved by the Ethics Committee in Clinical Research of The First Affiliated Hospital of Wenzhou Medical University (issuing no. 2019-128; Wenzhou, China).

Online analysis of the cancer genome atlas (TCGA) data. Kaplan-Meier analysis of TCGA database (https://cancergenome.nih.gov/abouttcga/overview) was performed by OncoLnc (version 2016: http://www.oncolnc.org/) using the lung adenocarcinoma (LUAD) dataset (21). Patients were divided into high miR-199a-5p expression (40\% upper percentile) and low miR-199a-5p expression (40\% lower percentile) groups.

Cell culture. The BEAS-2B normal human lung epithelial cell line and the H1299 and H460 NSCLC cell lines were purchased from American Type Culture Collection. Cells were cultured in RPMI-1640 medium (Gibco; Thermo Fisher Scientific, Inc.) supplemented with 10\% FBS (Gibco; Thermo Fisher Scientific, Inc.), $100 \mathrm{mg} / \mathrm{ml}$ streptomycin and $100 \mathrm{IU} / \mathrm{ml}$ penicillin in a $5 \% \mathrm{CO}_{2}$ atmosphere at $37^{\circ} \mathrm{C}$. Cell passage was performed every 2 days. 5-Aza-dC $(2 \mu \mathrm{M})$ purchased from Sigma-Aldrich; Merck KGaA was used to treat H1299 cells for $48 \mathrm{~h}$ at $37^{\circ} \mathrm{C}$.
Plasmids and mimic RNAs. miR-199a-5p or negative control (NC) mimic RNAs (miR-199a-5p mimics, 5'-CCCAGUGUU CAGACUACCUGUUC-3'; NC mimics, 5'-GGUUCGUAC GUACACUGUUCA-3', both $20 \mu \mathrm{M}$ ) were purchased from Shanghai GenePharma Co., Ltd. miR-199a-5p and AKAP1 overexpression plasmids (ov-miR-199a-5p and ov-AKAP1) were constructed using the pcDNA3.1 vector (both $500 \mathrm{ng} / \mu \mathrm{l}$, Invitrogen; Thermo Fisher Scientific, Inc.). The 3'UTR of the AKAP1 gene was amplified based on cDNA and then cloned into the pGL3 luciferase reporter vector (500 ng/ $\mu$; Promega Corporation) to obtain the AKAP1-3'UTR reporter plasmid (pGL3-AKAP1-3'UTR). A mutation reporter vector (pGL3-AKAP1-3'UTR-mut) was constructed via subcloning (the sequence ACU at the miR-199a-5p binding site was mutated to UGA). Transfection was performed using Lipofectamine ${ }^{\circledR}$ 3000 transfection reagent (Invitrogen; Thermo Fisher Scientific, Inc.) according to the manufacturer's protocols at $37^{\circ} \mathrm{C}$ for $24 \mathrm{~h}$. At $48 \mathrm{~h}$ after transfection, subsequent experiments were performed or G418 (Sigma-Aldrich; Merck KGaA) was used to screen $\mathrm{H} 1299$ cells with stable transfection.

Reverse transcription-quantitative PCR (RT-qPCR). Total RNA was extracted from cells and tissues using an RNAiso Plus kit (Takara Bio, Inc.). The RNA concentration was measured using a NanoDrop ND-1000 spectrophotometer (NanoDrop Technologies; Thermo Fisher Scientific, Inc.).

For mRNA, cDNA was reverse transcribed from $0.5 \mu \mathrm{g}$ mRNA using a PrimeScript RT reagent kit (Takara Bio, Inc.), and the following conditions were used for RT: $37^{\circ} \mathrm{C}$ for $30 \mathrm{~min}, 85^{\circ} \mathrm{C}$ for $15 \mathrm{sec}$. qPCR for AKAP1 (forward, $5^{\prime}-\mathrm{TCC}$ GTGGATAGCTGTTGCAG-3'; reverse, 5'-CTGCTTGCC AATTAGCCGAC-3') was performed using SYBR Green PCR Master Mix (Takara Bio, Inc.). The following thermocycling conditions were used: Initial denaturation at $95^{\circ} \mathrm{C}$ for $5 \mathrm{~min}$, followed by 40 cycles of $95^{\circ} \mathrm{C}$ for $45 \mathrm{sec}$, annealing at $55^{\circ} \mathrm{C}$ for $45 \mathrm{sec}$ and extension at $72^{\circ} \mathrm{C}$ for $1 \mathrm{~min}$. $\beta$-actin (forward, 5'-CTCCATCCTGGCCTCGCTGT-3'; reverse, 5'-GCTGTC ACCTTCACCGTTCC-3') was used as an internal control to normalize mRNA levels. The relative expression levels were calculated using the $2^{-\Delta \Delta \mathrm{Cq}}$ method (22).

For miRNA, a TaqMan microRNA RT kit (Applied Biosystems; Thermo Fisher Scientific, Inc.) was used for reverse transcription, and the following conditions for RT were used: $16^{\circ} \mathrm{C}$ for $30 \mathrm{~min}, 42^{\circ} \mathrm{C}$ for $30 \mathrm{~min}$ and $85^{\circ} \mathrm{C}$ for $5 \mathrm{~min}$. A TaqMan miRNA assay (Applied Biosystems; Thermo Fisher Scientific, Inc.) was used to measure the expression levels of mir-199a-5p (forward, 5'-AACCATGCCCAGTGTTCAGAC TA-3'; reverse, 5'-CAGTGCAGGGTCCGAGGT-3'). The following thermocycling conditions were used: Initial denaturation at $95^{\circ} \mathrm{C}$ for $10 \mathrm{~min}$, followed by 40 cycles of $95^{\circ} \mathrm{C}$ for $15 \mathrm{sec}$, annealing and extension at $60^{\circ} \mathrm{C}$ for $1 \mathrm{~min}$. U6 (forward, 5'-CTCGCTTCGGCAGCACA-3'; reverse, 5'-AACGCT TCACGAATTTGCGT-3') was used as an internal control to normalize miRNA levels. The relative expression levels were calculated using the $2^{-\Delta \Delta \mathrm{Cq}}$ method (22).

DNA sodium bisulfite conversion. Genomic DNA was extracted from NSCLC and para-carcinoma tissues using the phenol-chloroform technique. Bisulfite conversion was performed using an EpiJET Bisulfite Conversion Kit 
A

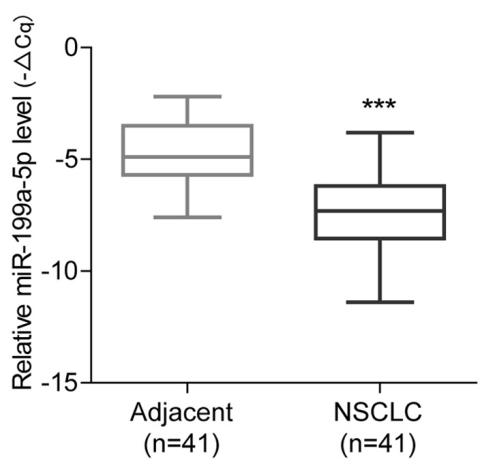

B

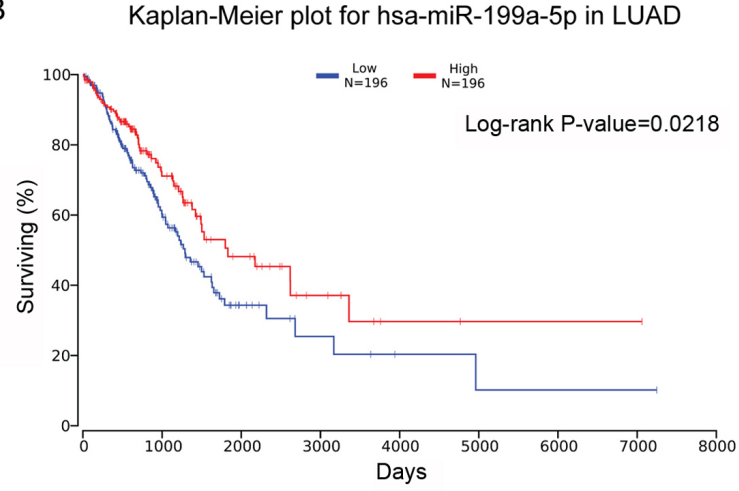

C

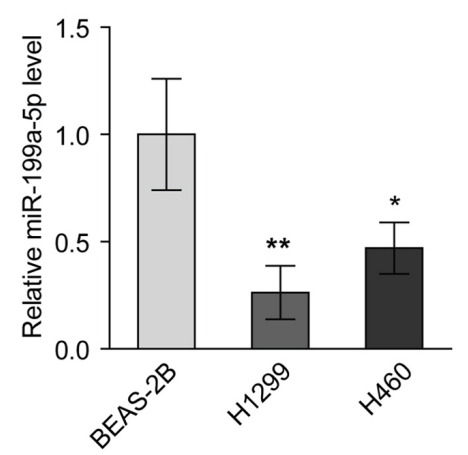

Figure 1. Expression levels of miR-199a-5p are decreased in NSCLC. (A) Expression levels of miR-199a-5p in 41 paired NSCLC and adjacent normal tissues were quantified using RT-qPCR using U6 as an internal control. $-\Delta \mathrm{Cq}$ [ $\Delta \mathrm{Cq}$ : Cq (miR-199a-5p)-Cq (U6)] represented the relative expression level. A paired t-test was used for analysis. ${ }^{* * *} \mathrm{P}<0.001$ vs. adjacent tissue. (B) Overall survival rates of patients with LUAD with high or low miR-199a-5p expression (high, $\mathrm{n}=196$; low, $\mathrm{n}=196$ ) were analyzed based on The Cancer Genome Atlas database using OncoLnc. (C) Expression levels of miR-199a-5p in BEAS-2B, H1299 and $\mathrm{H} 460$ cells were quantified via RT-qPCR using U6 as an internal control, and were normalized to BEAS-2B. One-way ANOVA with Tukey's post hoc test was used for analysis. "P $<0.05,{ }^{* *} \mathrm{P}<0.01$ vs. BEAS-2B cells. LUAD, lung adenocarcinoma; miR, microRNA; NSCLC, non-small cell lung cancer; RT-qPCR, reverse transcription-quantitative PCR.

(Thermo Fisher Scientific, Inc.) according to the manufacturer's protocols (incubation conditions: $98^{\circ} \mathrm{C}$ for $10 \mathrm{~min}, 60^{\circ} \mathrm{C}$ for $150 \mathrm{~min}$ ). Specific primers [Forward, 5'-TATATTTGGAAT TGTTTATAGT-3'; reverse, 5'-AAAAAAATATCTAACTCT TTAA-3' (23)] for the converted promoter region were used to generate the PCR product using Platinum ${ }^{\mathrm{TM}}$ Taq Green Hot Start DNA Polymerase (Invitrogen; Thermo Fisher Scientific, Inc.). Amplification was conducted by performing initial denaturation at $94^{\circ} \mathrm{C}$ for $5 \mathrm{~min}$, followed by 35 cycles of $94^{\circ} \mathrm{C}$ for $2 \mathrm{~min}, 94^{\circ} \mathrm{C}$ for $30 \mathrm{sec}, 60^{\circ} \mathrm{C}$ for $30 \mathrm{sec}$ and $72^{\circ} \mathrm{C}$ for $1 \mathrm{~min}$. PCR products were separated by $2 \%$ agarose gel and cloned into pGEM-Teasy (Promega Corporation) followed by sequencing with Sp6 primer. The dideoxy chain-termination method (Sanger method) was used to sequence the inserted fragments. The lollipop diagram from the sequencing data was generated by BiQ Analyzer v.2.0 (Max-Planck-Institut Informatik). Filled (black) circles corresponded to methylated Cs, and unfilled (white) circles corresponded to unmethylated Cs.

Western blotting. Whole cell protein was obtained using cold cell lysis RIPA buffer (cat. no. 20-188; EMD Millipore), and the total protein concentration was measured using a Bradford protein assay (cat. no. 23236; Bio-Rad Laboratories, Inc.). Equivalent amounts of protein $(30 \mu \mathrm{g} / \mathrm{lane})$ were separated via $10 \%$ SDS-PAGE and transferred to a nitrocellulose membrane
(Whatman plc; Cytiva). The membrane was blocked with $5 \%$ milk for $2 \mathrm{~h}$ at room temperature, and incubated at $4^{\circ} \mathrm{C}$ overnight with the following primary antibodies: AKAP1 (dilution, 1:1,000; cat. no. 5203; Cell Signaling Technology, Inc.) and GAPDH (dilution, 1:2,000; cat. no. sc-47724; Santa Cruz Biotechnology, Inc.). Subsequently, the membrane was incubated with an appropriate fluorescent secondary antibody (IRDye ${ }^{\circledR} 800 \mathrm{CW}$ - or IRDye ${ }^{\circledR} 680 \mathrm{RD}$-conjugated antibodies (dilution, 1:10,000; cat. nos. 926-32211 and 926-68070; LI-COR Biosciences) at room temperature for $1 \mathrm{~h}$. Subsequently, protein bands were detected using an Odyssey ${ }^{\circledR}$ infrared imaging system (LI-COR Biosciences). The obtained signals were converted into grayscale images using Application Software v.2.1.12 (LI-COR Biosciences).

Prediction of miR-199a-5p targets. TargetScan (TargetScan Release 3.1; http://www.targetscan.org) online prediction tools were used to predict miR-199a-5p target genes. Briefly, the default settings were adopted when setting up the run parameters. The gene was considered as a potential target gene of miR-199a-5p when its total context++ score was <-0.6.

Luciferase reporter assay. $\mathrm{H} 1299$ or $\mathrm{H} 460$ cells $\left(1 \times 10^{5}\right.$ cells/well) were plated onto a 24 -well plate. The following day, the cells in each well were co-transfected 

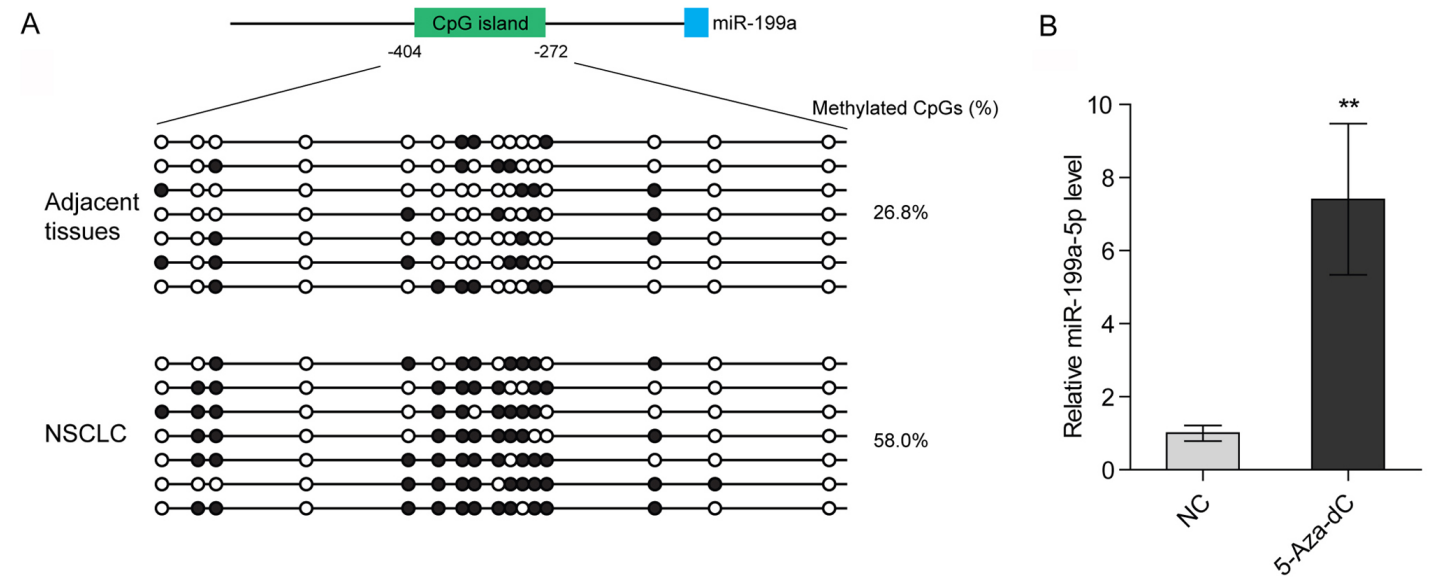

Figure 2. Hypermethylation of the promoter of miR-199a contributes to the low expression levels of miR-199a-5p in NSCLC. (A) Methylation levels of the miR-199a gene promoter in NSCLC and para-carcinoma tissues were determined using bisulphite DNA sequencing, showing the methylation status of CpGs in NSCLC and adjacent tissues. (B) Expression levels of miR-199a-5p in H1299 cells after 5-Aza-dC treatment were measured using reverse transcription-quantitative PCR using U6 as an internal control, and normalized to the NC group. An unpaired t-test was used for analysis. ${ }^{* *} \mathrm{P}<0.01 \mathrm{vs}$. NC group. 5-Aza-dC, 5-Aza-2'-deoxycytidine; miR, microRNA; NC, negative control; NSCLC, non-small cell lung cancer.

with $0.5 \mu \mathrm{g}$ firefly luciferase report plasmid (pGL3 with AKAP1 3'UTR) and $0.01 \mu \mathrm{g}$ Renilla luciferase report plasmid (pRL-SV40; Promega Corporation). The pRL-SV40 plasmid was used to standardize the transfection efficiency. Meanwhile, the cells in each well were also transfected with 40 pmol miR-199a-5p or negative control (NC) mimic RNAs (miR-199a-5p mimics, 5'-CCCAGUGUUCAGACU ACCUGUUC-3'; NC mimics, 5'-GGUUCGUACGUACAC UGUUCA-3', both $20 \mu \mathrm{M}$, Shanghai GenePharma Co., Ltd.). Transfection was performed using Lipofectamine ${ }^{\circledR} 3000$ transfection reagent (Invitrogen; Thermo Fisher Scientific, Inc.) according to the manufacturer's protocols at $37^{\circ} \mathrm{C}$. The cells were lysed using $100 \mu \mathrm{l}$ passive lysis buffer (Promega Corporation) at 2 days after transfection. The reporter activity was measured using the dual luciferase reporter assay system (cat. no. E1910; Promega Corporation) according to the manufacturer's protocols, and a luminometer (model no. LB9507; Titertek-Berthold). Briefly, $20 \mu$ c cell lysate was mixed with $100 \mu \mathrm{l}$ LAR II in a luminometer tube and then the tube was placed in the luminometer for the first measurement (firefly luciferase activity). Subsequently, $100 \mu \mathrm{l}$ Stop \& Glo ${ }^{\circledR}$ reagent was added and followed by gentle mixing. The tube was then placed in the luminometer again for the second measurement (Renilla luciferase activity). Relative luciferase activity was calculated by dividing the first measurement by the second.

Cell proliferation assay. Cell proliferation was analyzed using a Cell Counting Kit-8 (CCK-8; Dojindo Molecular Technologies, Inc.) assay. Cells $\left(3 \times 10^{3}\right)$ were added into each well of a 96-well plate. After the indicated time (12, 24, 36, 48 and $60 \mathrm{~h}), 10 \% \mathrm{CCK}-8$ reagent was added to each well and the cells were incubated for $2 \mathrm{~h}$. Measurements were obtained at a wavelength of $450 \mathrm{~nm}$ using a spectrophotometer (Bio-Rad Laboratories, Inc.).

EdU incorporation assay. The EdU (5-ethynyl-2-deoxyuridine) incorporation assay was used to represent DNA synthesis in cells. H1299 and H460 cells (5x104/well) were transfected with miR-199a-5p mimics or AKAP1 expression plasmids for
$48 \mathrm{~h}$ at $37^{\circ} \mathrm{C}$. Next, cells were washed 3 times with PBS, and then incubated in serum-free RPMI 1640 with $10 \mathrm{mM}$ EdU (Sigma-Aldrich; Merck KGaA) for $2 \mathrm{~h}$ at $37^{\circ} \mathrm{C}$. After extensive washing with PBS, cells were blocked with $10 \%$ FBS in PBS for $30 \mathrm{~min}$ at $37^{\circ} \mathrm{C}$. Incorporated EdU was detected by the fluorescent azide coupling reaction (Invitrogen; Thermo Fisher Scientific, Inc.). Images of the cells were captured with a fluorescence microscope (Nikon Ti-E) (magnification, x40) and analyzed by Image J v.1.4.3.67 (National Institutes of Health).

Colony formation assay. $\mathrm{H} 1299$ cells $\left(5 \times 10^{3}\right)$ were suspended in $2.5 \mathrm{ml}$ of $0.3 \%$ agar and then placed in each well of a 6 -well plate, which was pre-coated with $1.0 \mathrm{ml} 0.6 \%$ agar/well. Cells were cultured in an incubator at $37^{\circ} \mathrm{C}$ with $5 \% \mathrm{CO}_{2}$ and $95 \%$ air. The RPMI-1640 medium (Gibco; Thermo Fisher Scientific, Inc.) supplemented with $10 \%$ FBS (Gibco; Thermo Fisher Scientific, Inc.) was replaced with fresh medium every 3 days. After 21 days, the colonies were captured by a light microscope (Eclipse Ti), and at least 5 individual fields at magnification, x100 were chosen. Then colonies were counted using ImageJ software (version 1.4.3.67; National Institutes of Health).

Statistical analysis. SPSS v13.0 (SPSS, Inc.) and GraphPad Prism 5 (GraphPad Software, Inc.) were used for statistical analysis. Experimental data were presented as mean \pm SD from at least 3 independent experiments. An unpaired Student's t-test was used for comparisons between two independent groups. A paired t-test was used for comparisons between two paired groups. ANOVA (data with univariate change were analyzed using one-way with Tukey's post hoc test; data with multivariate change were analyzed using two-way with Sidak's post hoc test) was used for multiple group comparisons. A Pearson test was used for correlation analysis. $\mathrm{P}<0.05$ was considered to indicate a statistically significant difference.

\section{Results}

miR-199a-5p expression is decreased in NSCLC. $\mathrm{miR}-199 \mathrm{a}-5 \mathrm{p}$ has been reported to be a tumor suppressor in lung cancer $(9,13)$. 
A

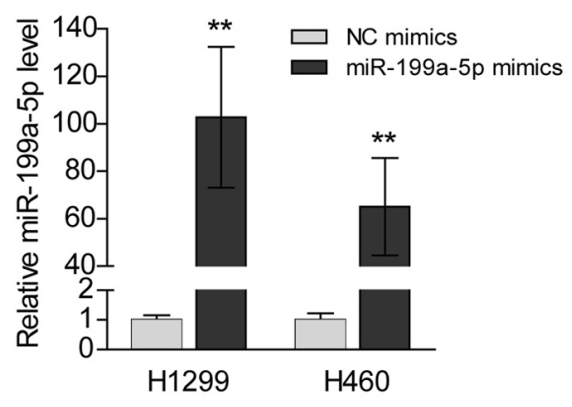

B

$\mathrm{H} 1299$

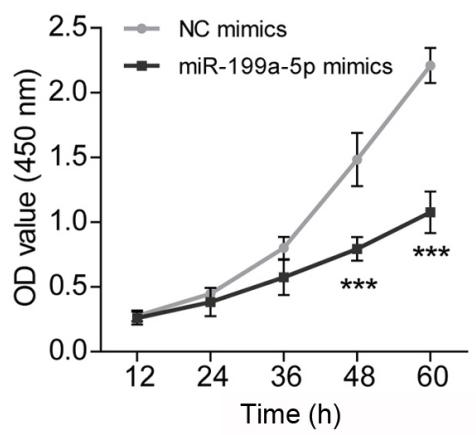

$\mathrm{H} 460$

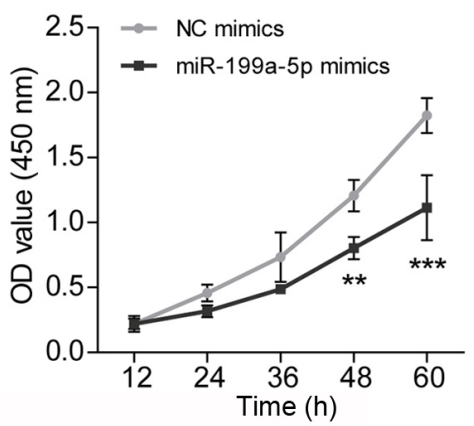

C
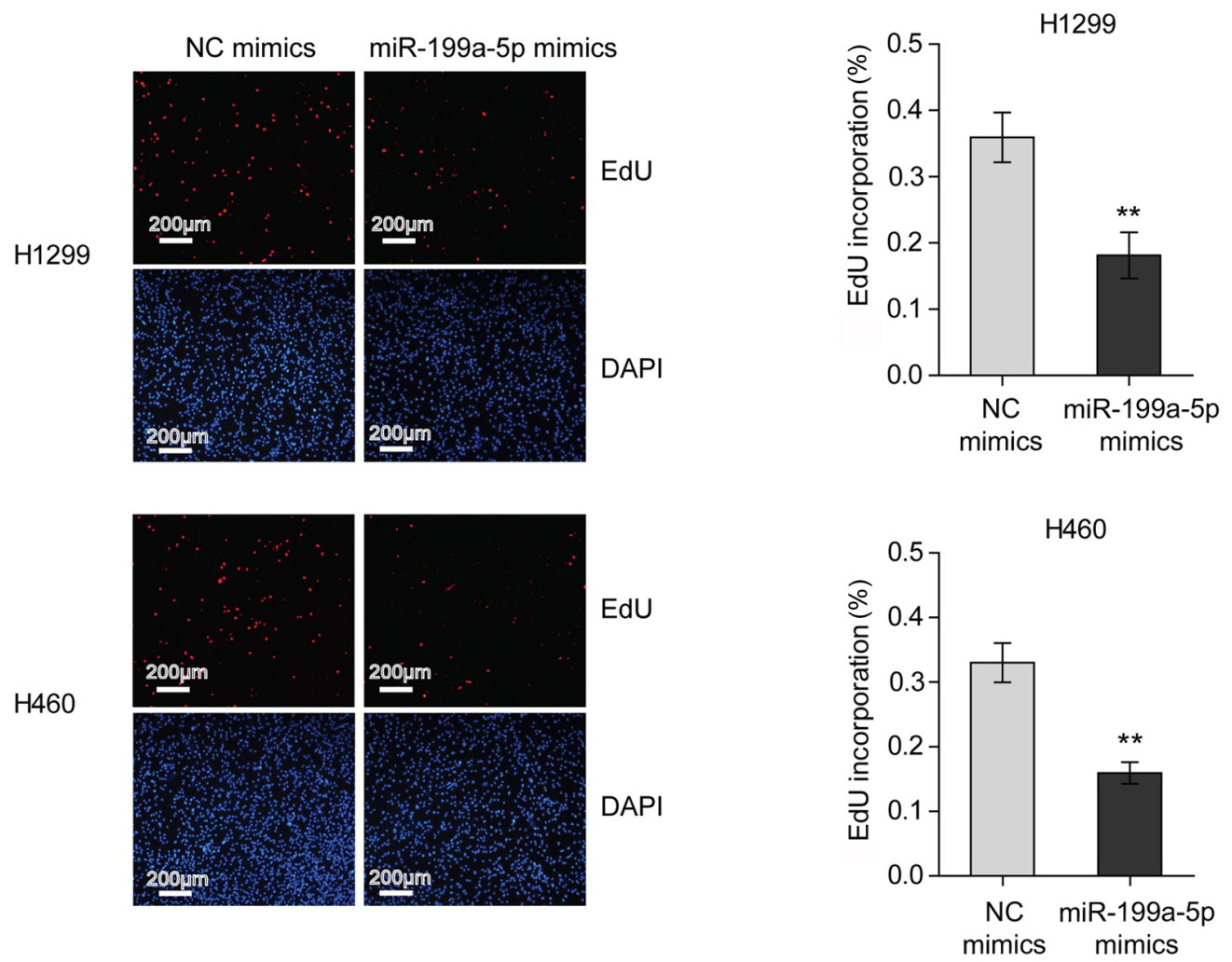

Figure 3. Transfection with miR-199a-5p mimics suppresses the proliferation of non-small cell lung cancer cells. (A) Transfection efficiency of miR-199a-5p mimics in $\mathrm{H} 1299$ and $\mathrm{H} 460$ cells was verified using reverse transcription-quantitative PCR using U6 as an internal control. miR-199a-5p expression was normalized to the NC mimics group. An unpaired t-test was used for analysis. (B) Cell proliferation was measured using a Cell Counting Kit-8 assay in H1299 and H460 cells transfected with miR-199a-5p mimics. Two-way ANOVA with Sidak's post hoc test was used for analysis. (C) DNA synthesis was measured using an EdU incorporation assay in H1299 and H460 cells transfected with miR-199a-5p mimics. The percentage of cells with EdU incorporation was measured using ImageJ software. An unpaired t-test was used for analysis. ${ }^{* *} \mathrm{P}<0.01,{ }^{* * *} \mathrm{P}<0.001 \mathrm{vs}$. NC mimics group. EdU, 5-ethynyl-2'-deoxyuridine; miR, microRNA; NC, negative control; OD, optical density.

The present study revealed that miR-199a-5p expression was downregulated in NSCLC tissues compared with para-carcinoma tissues (Fig. 1A). Furthermore, Kaplan-Meier analysis of a TCGA dataset using OncoLnc demonstrated that the overall survival rate of the patients with LUAD with high expression levels of miR-199a-5p was significantly increased compared with that of the patients with low miR-199a-5p expression (Fig. 1B). Consistently, the expression levels of miR-199a-5p were significantly lower in NSCLC cell lines (H1299 and H460) compared with in the BEAS-2B normal lung epithelial cell line (Fig. 1C). These findings suggested that the downregulation of miR-199a-5p expression was associated with NSCLC.
Hypermethylation of the promoter of the miR-199a gene contributes to the low expression levels of miR-199a-5p in $N S C L C$. Deng et al (23) reported that promoter methylation of the miR-199a gene suppresses miR-199a-3p in ovarian cancer. To determine whether the loss of miR-199a-5p in NSCLC also arises from promoter hypermethylation, bisulfite genomic sequencing was performed to analyze the methylation levels of the CpG island (-404/-272) located at the miR-199a promoter. It was revealed that the methylation levels of the $\mathrm{CpG}$ island were markedly higher in NSCLC tissues compared with in para-carcinoma tissues (Fig. 2A; Table SI). Additionally, treatment with a selective inhibitor of DNA methyltransferases 
A

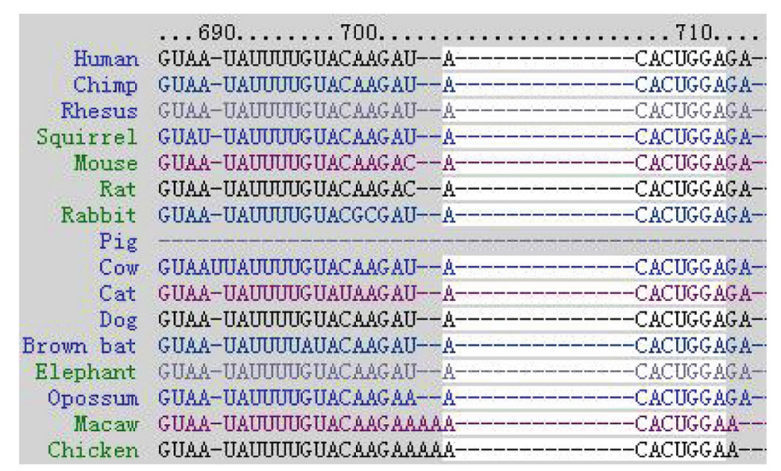

Position 706-713

of AKAP1 3' UTR

hsa-miR-199a-5p

uAUUUUGUACAAGAUACACUgGa

\|\|\|\| $\mid$

AKAP1 3' UTR MUT UAUUUUGUACAAGAUACUGAGGA
B
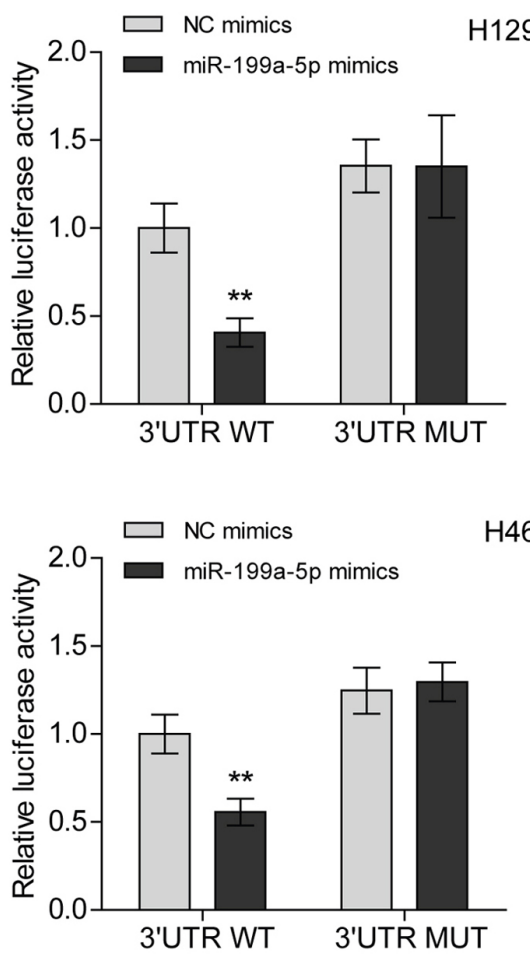

D

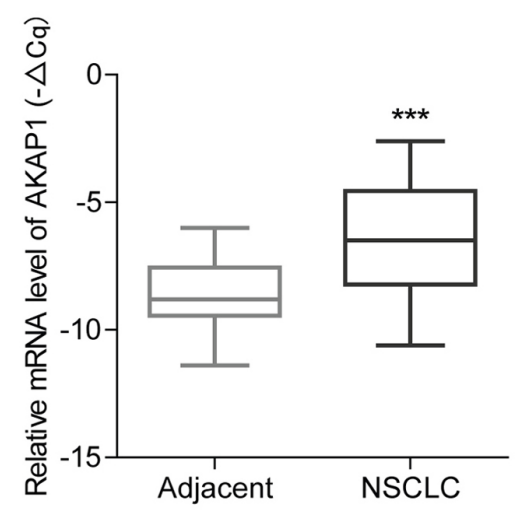

E

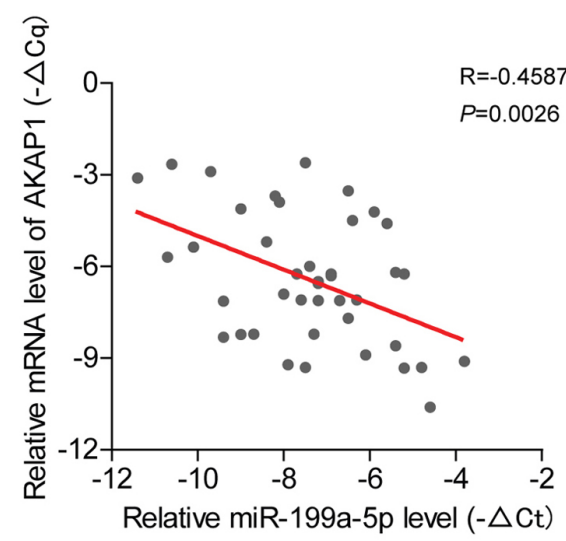

Figure 4. miR-199a-5p directly targets the mRNA of AKAP1 in NSCLC. (A) miR-199a-5p seed sequence complementary to the 3'UTR of AKAP1 mRNA was analyzed using TargetScan Human 7.2. (B) Luciferase activity was measured in H1299 and H460 cells co-transfected with the luciferase constructs containing the WT or MUT AKAP1 3'UTR, as well as miR-199a-5p mimics or NC mimics, and was normalized to the WT/NC mimics group. Two-way ANOVA with Sidak's post hoc test was used for analysis. ${ }^{* *} \mathrm{P}<0.01$ vs. WT/NC mimics group. (C) mRNA and protein expression levels of AKAP1 were analyzed using RT-qPCR and western blotting in $\mathrm{H} 1299$ and $\mathrm{H} 460$ cells transfected with miR-199a-5p mimics. mRNA expression levels were normalized to those of the NC mimics group. An unpaired t-test was used for analysis. "P<0.05 vs. NC mimics group. (D) mRNA expression levels of AKAP1 in 41 paired NSCLC and adjacent tissues were quantified via RT-qPCR, using $\beta$-actin as an internal control. $-\Delta \mathrm{Cq}[\Delta \mathrm{Cq}$ : Cq (AKAP1)-Cq ( $\beta$-actin)] represented the relative expression level. A paired t-test was used for analysis. ${ }^{* * *} \mathrm{P}<0.001$ vs. adjacent tissue. (E) Correlation between the mRNA expression levels of AKAP1 and miR-199a-5p in 41 NSCLC tissues. A Pearson test was used for analysis. AKAP1, A-kinase anchoring protein 1; miR, microRNA; MUT, mutant; NC, negative control; NSCLC, non-small cell lung cancer; RT-qPCR, reverse transcription-quantitative PCR; UTR, untranslated region; WT, wild-type. 

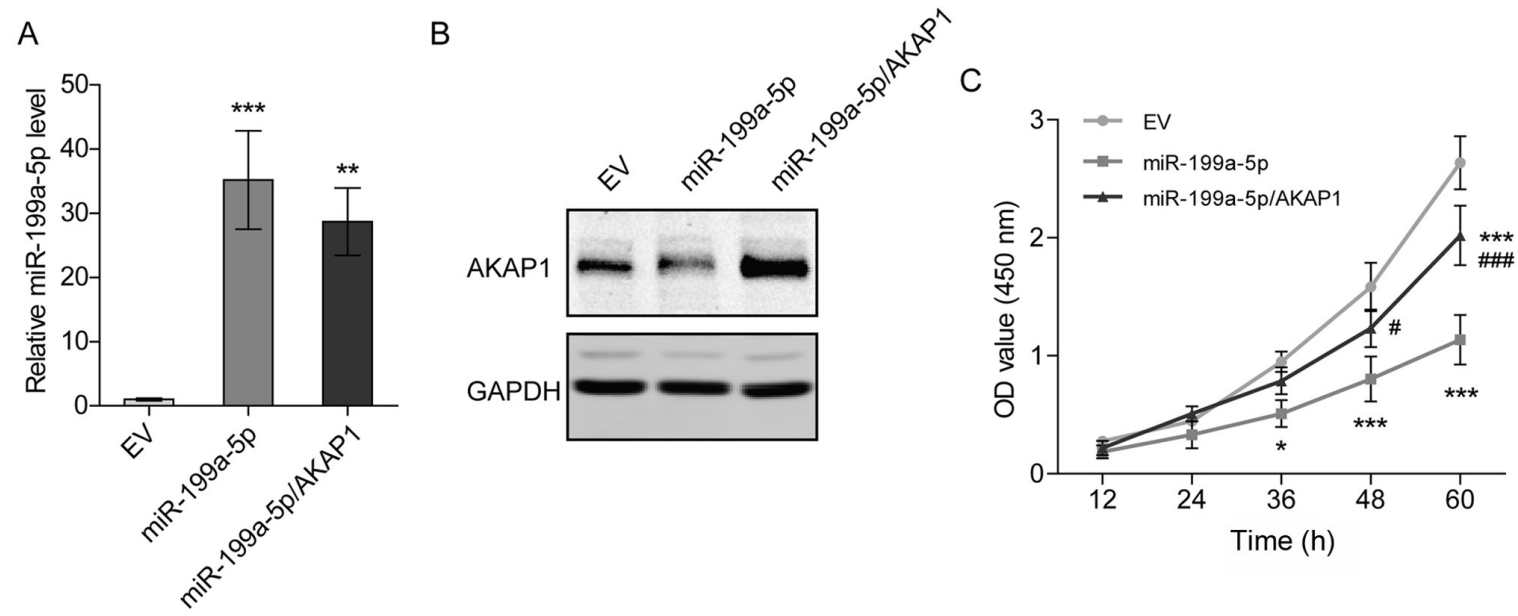

D
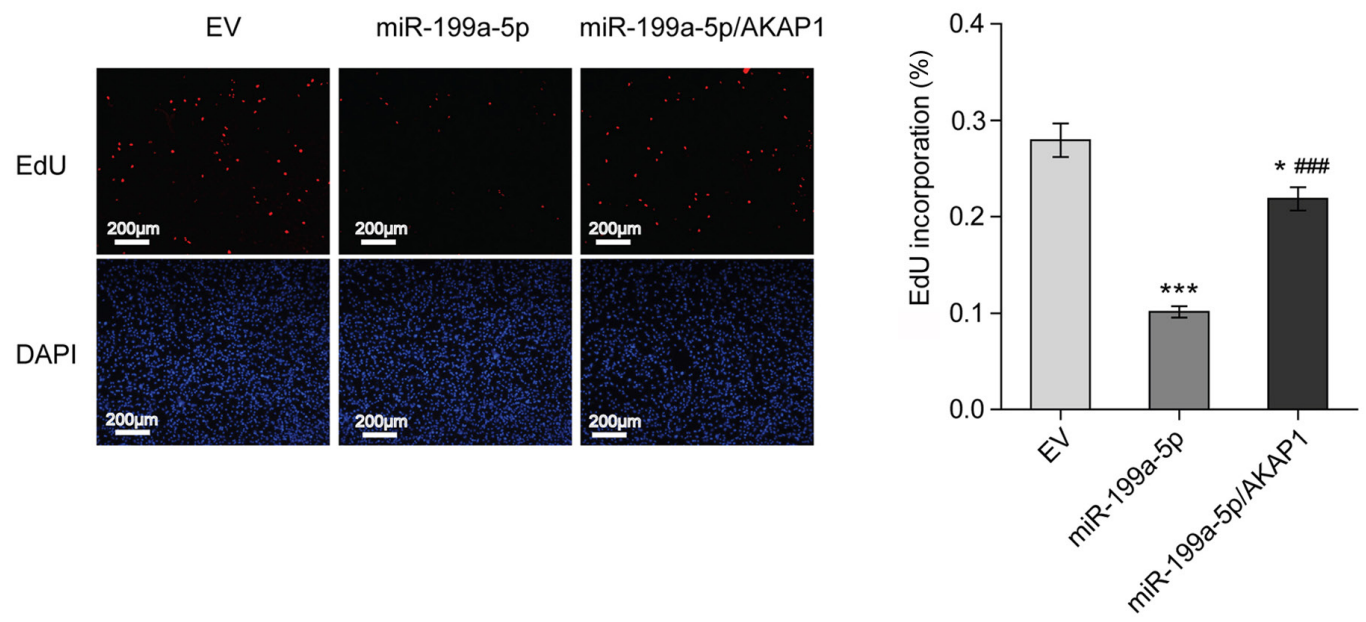

E
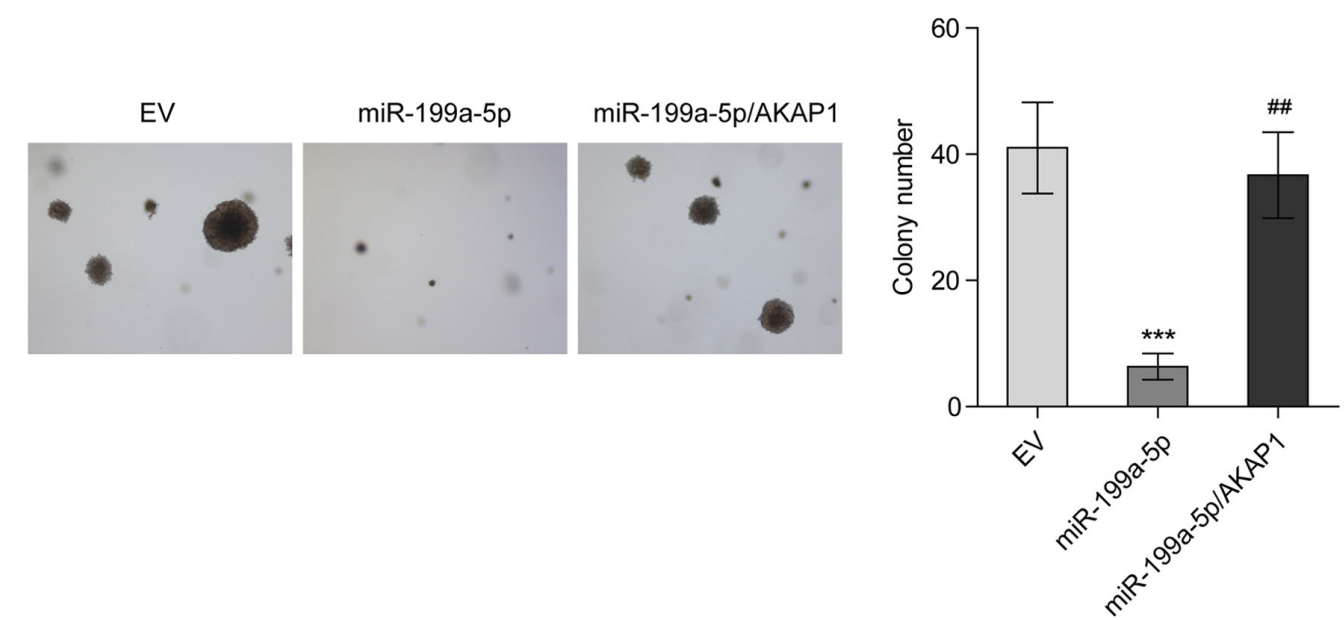

Figure 5. Overexpression of AKAP1 restores the proliferation of non-small cell lung cancer cells transfected with miR-199a-5p overexpression plasmid. Stable overexpression of (A) miR-199a-5p and (B) AKAP1 in H1299 cells was verified using reverse transcription-quantitative PCR and western blotting. miR-199a-5p expression was normalized to the EV group. One-way ANOVA with Tukey's post hoc test was used for analysis. (C) Cell proliferation was measured using a Cell Counting Kit-8 assay in H1299 cells stably overexpressing miR-199a-5p or AKAP1. Two-way ANOVA with Sidak's post hoc test was used for analysis. (D) DNA synthesis was measured using an EdU incorporation assay in H1299 cells stably overexpressing miR-199a-5p or AKAP1. The percentage of cells with EdU incorporation was measured using ImageJ software. One-way ANOVA with Tukey's post hoc test was used for analysis. (E) Colony formation of H1299 cells stably overexpressing miR-199a-5p or AKAP1. Colony numbers were counted using a microscope (magnification, x100). One-way ANOVA with Tukey's post hoc test was used for analysis. ${ }^{*} \mathrm{P}<0.05,{ }^{* *} \mathrm{P}<0.01$ and ${ }^{* * * *} \mathrm{P}<0.001$ vs. EV group; ${ }^{\# P} \mathrm{P}<0.05,{ }^{\# \#} \mathrm{P}<0.01$ and ${ }^{\# \# \#} \mathrm{P}<0.001$ vs. miR-199a-5p group. AKAP1, A-kinase anchoring protein 1; EdU, 5-ethynyl-2'-deoxyuridine; EV, empty vector; miR, microRNA; OD, optical density.

[5-Aza-2'-deoxycytidine (5-Aza-dC)] significantly increased the expression levels of miR-199a-5p in H1299 cells (Fig. 2B).
These data indicated that promoter hypermethylation may lead to the loss of miR-199a-5p in NSCLC. 
Transfection with miR-199a-5p mimics suppresses the proliferation of NSCLC cells. To investigate the role of miR-199a-5p in NSCLC development, the proliferation of H1299 and H460 cells transfected with miR-199a-5p mimics was measured. The transfection efficiency was verified by RT-qPCR (Fig. 3A). The results of the CCK- 8 assays demonstrated that the proliferation rates of H1299 and H460 cells were decreased following miR-199a-5p mimics transfection (Fig. 3B). Furthermore, it was revealed that the transfection of miR-199a-5p mimics interrupted 5-ethynyl-2'-deoxyuridine (EdU) incorporation, indicating that miR-199a-5p inhibited the DNA synthesis of NSCLC cells (Fig. 3C).

miR-199a-5p directly targets AKAP1 mRNA in NSCLC. To understand the mechanism underlying the antitumor function of miR-199a-5p in NSCLC, TargetScan was used to predict the potential transcripts targeted by miR-199a-5p. The seed sequence of miR-199a-5p was complementary to the 3'UTR of AKAP1 mRNA and is highly conserved among different species (Fig. 4A). Recently, the unique involvement of AKAP1 in tumor growth has been reviewed (14). In the present study, the luciferase assay results demonstrated that miR-199a-5p inhibited the activity of the reporter with wild-type 3'UTR of AKAP1 mRNA, but was not able to change the activity of the reporter when the miR-199a-5p binding site was mutated (Fig. 4B). Consistently, the mRNA and protein expression levels of AKAP1 were decreased following miR-199a-5p mimic transfection in $\mathrm{H} 1299$ and H460 cells (Fig. 4C). Since miR-199a-5p expression was induced by 5 -Aza-dC treatment, the expression levels of AKAP1 were measured after 5-Aza-dC treatment. The results indicated that 5-Aza-dC decreased the mRNA and protein expression levels of AKAP1 (Fig. S1). In contrast to miR-199a-5p, clinical analysis demonstrated that the mRNA expression levels of AKAP1 were significantly higher in NSCLC tissues compared with in para-carcinoma tissues (Fig. 4D). Furthermore, the mRNA expression levels of AKAP1 were negatively correlated with miR-199a-5p expression in NSCLC tissues (Fig. 4E). Collectively, these findings suggested that miR-199a-5p may downregulate AKAP1 in NSCLC.

Overexpression of AKAP1 restores the proliferation of NSCLC cells transfected with miR-199a-5p mimics. To verify whether miR-199a-5p represses the proliferation of H1299 cells by downregulating AKAP1, H1299 cells stably overexpressing miR-199a-5p and AKAP1 were constructed (Figs. 5A, B and $\mathrm{S} 2)$. Compared with the control group (EV), a suppression of cell proliferation and DNA synthesis was observed in H1299 cells stably overexpressing miR-199a-5p after $36 \mathrm{~h}$ of culturing, while overexpression of AKAP1 partially rescued cell proliferation and DNA synthesis (Fig. 5C and D). Additionally, overexpression of miR-199a-5p decreased the colony formation ability of H1299 cells, and overexpression of AKAP1, to a certain extent, recovered this attenuated colony formation (Fig. 5E). These results suggested that miR-199a-5p inhibited the proliferation of NSCLC cells by targeting AKAP1.

\section{Discussion}

The present study demonstrated that promoter hypermethylation inhibited the transcription of miR-199a-5p, which decreased the expression levels of AKAP1 by targeting AKAP1 mRNA. Furthermore, increased AKAP1 expression promoted the proliferation and colony formation of NSCLC cells.

Abnormal regulation of miRNAs serves an important role in the occurrence and development of lung cancer (24). The inhibition of miRNA biosynthesis has been observed in lung cancer (24). For example, Karube et al (25) reported that the expression levels of Dicer, a key endoribonuclease in miRNA-mediated gene silencing was decreased in NSCLC and low Dicer expression was associated with poor prognosis. Additionally, the results of animal experiments have demonstrated that conditional knockout of Dicer promotes tumor development in K-RAS-induced lung cancer (26). In rats, the tobacco carcinogen nicotine-derived nitrosamine ketone induces the dysregulation of various miRNAs (27). In addition, changes in the miRNA expression profile have been detected in precancerous lesions of the bronchial epithelium (28). However, it is difficult to identify specific miRNA markers for NSCLC due to tumor heterogeneity and different analysis methods. Wang et al (29) retrospectively analyzed four studies comparing the miRNA expression profiles between NSCLC and corresponding para-cancerous tissues; however, the differentially expressed miRNAs were different in each study.

The present study demonstrated that low expression levels of miR-199a-5p in NSCLC were associated with a poor survival, suggesting that miR-199a-5p may be a potential miRNA marker for the prediction of the prognosis of patients with NSCLC. Previously, Sanfiorenzo et al (30) reported that low expression levels of miR-199a-5p in the plasma are associated with poor survival of patients with NSCLC. Recently, it was revealed that miR-199a-5p could increase the doxorubicin sensitivity of NSCLC (31). Therefore, miR-199a-5p may function as a crucial tumor suppressor miRNA for NSCLC.

Several studies have revealed that long non-coding RNAs promote the proliferation of NSCLC via sponging of miR-199a-5p $(32,33)$. In addition, hypermethylation of the $\mathrm{CpG}$ island has been identified to be closely associated with the abnormal miRNA expression profiles in tumors (34). In total $\sim 1 / 2$ of all miRNAs are regulated by the CpG island (35). Previous studies have reported that the altered status of DNA methylation leads to the abnormal regulation of miRNA expression in NSCLC (36-38). The miR-199a gene is located at two different chromosomes (miR-199a1 is located at chromosome 19 and miR-199a2 is located at chromosome 1) (23). Therefore, the present study first analyzed the distribution of $\mathrm{CpG}$ islands at the promoter regions of the two gene loci using bioinformatics software, and identified that there was a CG-rich region (-404/-272) upstream of the transcription start site of miR-199a1, while no potential $\mathrm{CpG}$ island was found at the miR-199a2 promoter, which was consistent with a previous study (23). Therefore, the present study only analyzed the transcriptional regulation of the miR-199a1 gene. Bisulfite sequencing demonstrated that the methylation levels of the $\mathrm{CpG}$ island at the miR-199a promoter were increased in NSCLC compared with in para-cancerous tissues. Furthermore, treatment with a DNA methyltransferase inhibitor increased the expression levels of miR-199a-5p in NSCLC cells. These findings suggested that promoter hypermethylation suppressed miR-199a-5p expression in NSCLC.

Deng et al (23) revealed that knockdown of DNA methyltransferase $3 \alpha$ (DNMT3A) reverses the hypermethylation 
of the miR-199a gene in ovarian cancer. Furthermore, DNMT3A is highly expressed in various cancer types, including NSCLC, and can mediate the epigenetic silencing of tumor suppressor genes (39). Therefore, it was hypothesized that the promoter hypermethylation of the miR-199a gene may also be induced by aberrantly expressed DNMT3A in NSCLC.

The present study demonstrated that miR-199a-5p inhibited the proliferation of NSCLC cells by targeting AKAP1. Rinaldi et al (17) reported that AKAP1 promotes glioblastoma (GBM) growth by enhancing the mTOR signaling pathway. In addition, these authors observed that knockdown of AKAP1 severely impaired the oxidative metabolism of GBM cells In general, high oxidative phosphorylation (OXPHOS) is adopted to promote tumor growth $(40,41)$, and AKAP1 stimulates mitochondrial respiration (42). Therefore, deregulated AKAP1 may enhance OXPHOS to induce the proliferation of NSCLC cells.

In conclusion, the present study indicated that promoter hypermethylation caused the downregulation of miR-199a-5p expression in NSCLC, and miR-199a-5p was identified as a novel regulator of AKAP1 expression influencing NSCLC proliferation. Therefore, the present findings may provide a prognostic miRNA marker for patients with NSCLC.

\section{Acknowledgements}

Not applicable.

\section{Funding}

The present study was supported by the Program of Wenzhou Science and Technology Bureau (grant no. Y20190113).

\section{Availability of data and materials}

All data generated or analyzed during this study are included in this published article.

\section{Authors' contributions}

NY and LJ conceived and designed the study. NY, YFL, TZ and YXL performed the experiments and wrote the manuscript. NY, ZC and XZ analyzed and interpreted the data. NY, $\mathrm{XZ}$ and LJ confirmed the authenticity of all the raw data. NY, $\mathrm{XZ}$ and LJ drafted the manuscript. All other authors helped to draft the manuscript. All authors read and approved the final manuscript.

\section{Ethics approval and consent to participate}

Experiments using human tissues were approved by the Ethics Committee in Clinical Research of the First Affiliated Hospital of Wenzhou Medical University (issuing no. 2019-128; Wenzhou, China) and written informed consent was obtained from all patients.

\section{Patient consent for publication}

Not applicable.

\section{Competing interests}

The authors declare that they have no competing interests.

\section{References}

1. Allemani C, Weir HK, Carreira H, Harewood R, Spika D, Wang XS, Bannon F, Ahn JV, Johnson CJ, Bonaventure A, et al: Global surveillance of cancer survival 1995-2009: Analysis of individual data for $25,676,887$ patients from 279 population-based registries in 67 countries (CONCORD-2). Lancet 385: 977-1010, 2015.

2. Riaz SP, Lüchtenborg M, Coupland VH, Spicer J, Peake MD and Møller H: Trends in incidence of small cell lung cancer and all lung cancer. Lung Cancer 75: 280-284, 2012.

3. Toschi L, Cappuzzo F and Jänne PA: Evolution and future perspectives in the treatment of locally advanced non-small cell lung cancer. Ann Oncol 18 (Suppl 9): ix150-ix155, 2007.

4. Zhong J, Huang R, Su Z, Zhang M, Xu M, Gong J, Chen N, Zeng H, Chen X and Zhou Q: Downregulation of miR-199a-5p promotes prostate adeno-carcinoma progression through loss of its inhibition of HIF-1 $\alpha$. Oncotarget 8: 83523-83538, 2017.

5. Turashvili G, Lightbody ED, Tyryshkin K, SenGupta SK, Elliott BE, Madarnas Y, Ghaffari A, Day A and Nicol CJB: Novel prognostic and predictive microRNA targets for triple-negative breast cancer. FASEB J: May 29, 2018 (Epub ahead of print).

6. Ghosh A, Dasgupta D, Ghosh A, Roychoudhury S, Kumar D, Gorain M, Butti R, Datta S, Agarwal S, Gupta S, et al: MiRNA199a-3p suppresses tumor growth, migration, invasion and angiogenesis in hepatocellular carcinoma by targeting VEGFA, VEGFR1, VEGFR2, HGF and MMP2. Cell Death Dis 8: e2706, 2017.

7. Gadducci A, Sergiampietri C, Lanfredini N and Guiggi I: Micro-RNAs and ovarian cancer: The state of art and perspectives of clinical research. Gynecol Endocrinol 30: 266-271, 2014.

8. Ye H, Pang L, Wu Q, Zhu Y, Guo C, Deng Y and Zheng X: A critical role of mir-199a in the cell biological behaviors of colorectal cancer. Diagn Pathol 10: 65, 2015.

9. Ahmadi A, Khansarinejad B, Hosseinkhani S, Ghanei M and Mowla SJ: miR-199a-5p and miR-495 target GRP78 within UPR pathway of lung cancer. Gene 620: 15-22, 2017.

10. Hu Y, Liu J, Jiang B, Chen J, Fu Z, Bai F, Jiang J and Tang Z: MiR-199a-5p loss up-regulated DDR1 aggravated colorectal cancer by activating epithelial-to-mesenchymal transition related signaling. Dig Dis Sci 59: 2163-2172, 2014.

11. Guo W, Qiu Z, Wang Z, Wang Q, Tan N, Chen T, Chen Z, Huang S, $\mathrm{Gu}$ J, Li J, et al: miR-199a-5p is negatively associated with malignancies and regulates glycolysis and lactate production by targeting hexokinase 2 in liver cancer. Hepatology 62: 1132-1144, 2015.

12. Li W, Wang H,Zhang J,Zhai L, Chen W and Zhao C: miR-199a-5p regulates $\beta 1$ integrin through Ets-1 to suppress invasion in breast cancer. Cancer Sci 107: 916-923, 2016.

13. Li Y, Wang D, Li X, Shao Y, He Y, Yu H and Ma Z: MiR-199a-5p suppresses non-small cell lung cancer via targeting MAP3K11. J Cancer 10: 2472-2479, 2019.

14. Liu Y, Merrill RA and Strack S: A-kinase anchoring protein 1: Emerging roles in regulating mitochondrial form and function in health and disease. Cells 9: 298, 2020.

15. Yu J, Zhang Y, Zhou D, Wu J and Luo R: Higher expression of A-kinase anchoring-protein 1 predicts poor prognosis in human hepatocellular carcinoma. Oncol Lett 16: 131-136, 2018.

16. Li B, Wang W, Li Z, Chen Z, Zhi X, Xu J, Li Q, Wang L, Huang X, Wang L, et al: MicroRNA-148a-3p enhances cisplatin cytotoxicity in gastric cancer through mitochondrial fission induction and cyto-protective autophagy suppression. Cancer Lett 410: 212-227, 2017.

17. Rinaldi L, Sepe M, Delle Donne R, Conte K, Arcella A, Borzacchiello D, Amente S, De Vita F, Porpora M, Garbi C, et al: Mitochondrial AKAP1 supports mTOR pathway and tumor growth. Cell Death Dis 8: e2842, 2017.

18. Rami-Porta R, Bolejack V, Crowley J, Ball D, Kim J, Lyons G, Rice T, Suzuki K, Thomas CF Jr, Travis WD, et al: The IASLC Lung cancer staging project: Proposals for the revisions of the $\mathrm{T}$ descriptors in the forthcoming eighth edition of the TNM classification for lung cancer. J Thorac Oncol 10: 990-1003, 2015.

19. Travis WD: The 2015 WHO classification of lung tumors. Pathologe 35 (Suppl 2): 188, 2014. 
20. Chen K, Chen H, Yang F, Sui X, Li X and Wang J: Validation of the eighth edition of the TNM staging system for lung cancer in 2043 surgically treated patients with non-small-cell lung cancer. Clin Lung Cancer 18: e457-e466, 2017.

21. Cancer Genome Atlas Research Network: Comprehensive molecular profiling of lung adenocarcinoma. Nature 511: 543-550, 2014.

22. Livak KJ and Schmittgen TD: Analysis of relative gene expression data using real-time quantitative PCR and the 2(-Delta Delta C(T)) method. Methods 25: 402-408, 2001.

23. Deng Y, Zhao F, Hui L, Li X, Zhang D, Lin W, Chen Z and Ning Y: Suppressing miR-199a-3p by promoter methylation contributes to tumor aggressiveness and cisplatin resistance of ovarian cancer through promoting DDR1 expression. J Ovarian Res 10: 50, 2017.

24. Iqbal MA, Arora S, Prakasam G, Calin GA and Syed MA: MicroRNA in lung cancer: Role, mechanisms, pathways and therapeutic relevance. Mol Aspects Med 70: 3-20, 2019.

25. Karube Y, Tanaka H, Osada H, Tomida S, Tatematsu Y, Yanagisawa K, Yatabe Y, Takamizawa J, Miyoshi S, Mitsudomi T and Takahashi T: Reduced expression of Dicer associated with poor prognosis in lung cancer patients. Cancer Sci 96: 111-115, 2005.

26. Kumar MS, Lu J, Mercer KL, Golub TR and Jacks T: Impaired microRNA processing enhances cellular transformation and tumorigenesis. Nat Genet 39: 673-677, 2007.

27. Kalscheuer S, Zhang X, Zeng Y and Upadhyaya P: Differential expression of microRNAs in early-stage neoplastic transformation in the lungs of F344 rats chronically treated with the tobacco carcinogen 4-(methylnitrosamino)-1-(3-pyridyl)-1-butanone. Carcinogenesis 29: 2394-2399, 2008.

28. Mascaux C, Laes JF, Anthoine G, Haller A, Ninane V, Burny A and Sculier JP: Evolution of microRNA expression during human bronchial squamous carcinogenesis. Eur Respir J 33: 352-359, 2009

29. Wang QZ, Xu W, Habib N and Xu R: Potential uses of microRNA in lung cancer diagnosis, prognosis, and therapy. Curr Cancer Drug Targets 9: 572-594, 2009 .

30. Sanfiorenzo C, Ilie MI, Belaid A, Barlési F, Mouroux J, Marquette $\mathrm{CH}$, Brest $\mathrm{P}$ and Hofman P: Two panels of plasma microRNAs as non-invasive biomarkers for prediction of recurrence in resectable NSCLC. PLoS One 8: e54596, 2013.

31. Jin Y, Wang $\mathrm{H}$, Zhu Y, Feng $\mathrm{H}$, Wang $\mathrm{G}$ and Wang S: miR-199a-5p is involved in doxorubicin resistance of non-small cell lung cancer (NSCLC) cells. Eur J Pharmacol 878: 173105, 2020.

32. Hua Q, Jin M, Mi B, Xu F, Li T, Zhao L, Liu J and Huang G: LINC01123, a c-Myc-activated long non-coding RNA, promotes proliferation and aerobic glycolysis of non-small cell lung cancer through miR-199a-5p/c-Myc axis. J Hematol Oncol 12: 91, 2019.
33. Wang C, Han C, Zhang Y and Liu F: LncRNA PVT1 regulate expression of HIF1 $\alpha$ via functioning as ceRNA for miR-199a-5p in non-small cell lung cancer under hypoxia. Mol Med Rep 17: 1105-1110, 2018

34. Suzuki H, Maruyama R, Yamamoto E and Kai M: DNA methylation and microRNA dysregulation in cancer. Mol Oncol 6: 567-578, 2012.

35. Weber B, Stresemann C, Brueckner B and Lyko F: Methylation of human microRNA genes in normal and neoplastic cells. Cell Cycle 6: 1001-1005, 2007.

36. Zhang J, Fu J, Pan Y, Zhang X and Shen L: Silencing of miR-1247 by DNA methylation promoted non-small-cell lung cancer cell invasion and migration by effects of STMN1. Onco Targets Ther 9: 7297-7307, 2016.

37. Liu C, Lv D, Li M, Zhang X, Sun G, Bai Y and Chang D: Hypermethylation of miRNA-589 promoter leads to upregulation of HDAC5 which promotes malignancy in non-small cell lung cancer. Int J Oncol 50: 2079-2090, 2017.

38. Xia W, Chen Q, Wang J, Mao Q, Dong G, Shi R, Zheng Y, $\mathrm{Xu}$ L and Jiang F: DNA methylation mediated silencing of microRNA-145 is a potential prognostic marker in patients with lung adenocarcinoma. Sci Rep 5: 16901, 2015.

39. Lin RK, Hsu HS, Chang JW, Chen CY, Chen JT and Wang YC: Alteration of DNA methyltransferases contributes to $5^{\prime} \mathrm{CpG}$ methylation and poor prognosis in lung cancer. Lung Cancer 55: 205-213, 2007.

40. Martinez-Outschoorn UE, Pestell RG, Howell A, Tykocinski ML, Nagajyothi F, Machado FS, Tanowitz HB, Sotgia F and Lisanti MP: Energy transfer in 'parasitic' cancer metabolism: Mitochondria are the powerhouse and Achilles' heel of tumor cells. Cell Cycle 10: 4208-4216, 2011.

41. Sotgia F, Martinez-Outschoorn UE and Lisanti MP: Cancer metabolism: New validated targets for drug discovery. Oncotarget 4: 1309-1316, 2013.

42. Livigni A, Scorziello A, Agnese S, Adornetto A, Carlucci A, Garbi C, Castaldo I, Annunziato L, Avvedimento EV and Feliciello A: Mitochondrial AKAP121 links cAMP and src signaling to oxidative metabolism. Mol Biol Cell 17: 263-271, 2006.

(i) $($ ) This work is licensed under a Creative Commons Attribution-NonCommercial-NoDerivatives 4.0 International (CC BY-NC-ND 4.0) License. 